\title{
An Improved Adaptive Genetic Algorithm
}

\author{
Song xudong \\ Software Institute; Network control \& Intelligent \\ System Center \\ Dalian Jiaotong University \\ Dalian, Liaoning, China \\ xudongsong@126.com
}

\author{
Xiao yunlong \\ Software Institute; Network control \& Intelligent \\ System Center \\ Dalian Jiaotong University \\ Dalian, Liaoning, China \\ williamxyl1989@126.com
}

\begin{abstract}
In this paper, we proposed a new adaptive genetic algorithm based hybrid coding and elitist strategy. The high fitness individuals was used to perform decimal coding to improve the search rate based on original adaptive genetic algorithm, as well as the criteria of poor individuals about elitist strategy, rather than random replacement or replacing the worst individual. Experimental results show that this method is effective and reliable.
\end{abstract}

Keywords-Genetic adaptive algorithm; hybrid coding; elitist strategy

\section{INTRODUCTION}

Genetic Algorithm [1] is a random search method evolved by the laws of evolution of the biosphere (survival of the fittest, survival of the fittest genetic mechanism).Calculation of the process of biological evolution model to simulate natural selection and genetic mechanism of Darwin's theory of biological evolution, is a method by simulating the natural evolution of the process of searching the optimal solution. It is first proposed in 1975 by the Holland Professor, the main characteristic is to operate directly on the structure of the object: no derivation and limitation of function continuity, the inherent implicit parallelism and better global optimization capability, the use of probabilistic optimization method, the ability of obtaining automatically and guidance to optimize the search space, adjusting the search direction adaptively, no rules needed to be determined. This property of the genetic algorithm has been widely used in combinatorial optimization, machine learning, signal processing, adaptive control and artificial life. It is a key technology in the modern about intelligent computing.

In the Standard Genetic Algorithm (SGA), operating parameter is artificially set in advance, which is poor in both the application of adaptive genetic algorithm, and the set of operating parameters at reducing the operating efficiency of the algorithm. In the calculation process, it also easily leads to the generation of the "premature" phenomenon. Because of these shortcomings of the SGA, Srinivas et al proposed the concept of adaptive genetic algorithm (AGA ), the advantage is the crossover and mutation probability adaptively adjust in algorithm[2]. This method improve the speed of convergence of the genetic algorithm to some extent, however, this algorithm still has no effective ways to avoid the phenomenon of "premature". The defects and deficiencies of AGA prompted a growing number of scientists to study and discussion of adaptive genetic algorithm.

This article perform decimal variation to high fitness individuals in order to retain their properties at the same time increase the optimization chance based on adaptive genetic algorithm. In elitist strategy, by adding a new judgment condition of poor individuals to avoid premature obsolescence of some the individual contemporary poor fitness, but still useful evolutionary potential of individuals.

\section{RELATED TECHNOLOGIES}

\section{A. Overview of Genetic Algorithm}

Genetic Algorithm is essentially a reproduction, the detection and evaluation of the iterative algorithm, it is a random search algorithm, its core content is the parameter encoding, set the initial population, fitness function design, genetic operators design, crossover and mutation operator settings [3].

The basic process of Standard Genetic Algorithm is as follows:

a. Initialization: Set the evolution algebra Counters $t=0$, set the maximum evolution algebra $\mathrm{T}, \mathrm{M}$ individuals randomly generated as the initial population $\mathrm{P}(0)$.

b. Individual Evaluation: Calculate the fitness of individuals in groups $\mathrm{P}(\mathrm{t})$.

c. Select computing: the select operator acting in groups. The purpose of select is inherited optimization individuals to the next generation or new individual paired cross then inherited to the next generation. The operation is based on the assessment the individual's fitness in the groups.

d. Crossover operation: the crossover operator acting in groups. The so-called cross-refers to the part of the structure of the two parent individuals replace restructuring and generate new individual operation.

e. Mutation operation: the mutation operator acting on the groups. That changes the value of some of the individual string in groups.

Get next generation of groups $\mathrm{P}(\mathrm{t}+1)$ after the groups of $\mathrm{P}(\mathrm{t})$ after the selection, crossover and mutation operation. f. The conditional of terminate: if $t=T$, by the evolutionary process as the optimal solution with the maximum fitness individual output, terminate the calculation. 
This shows that the selection operator, crossover operator and mutation operator plays a central role in the Genetic Algorithm.

\section{B. Adaptive Genetic Algorithm}

In the standard genetic algorithm, the crossover probability $\mathrm{P}_{c}$ and mutation probability $\mathrm{P}_{\mathrm{m}}$ is an artificial set of fixed values, their value has a significant impact on the performance of the algorithm, such as optimization ability and convergence rate. Crossover probability determines the pace of new individuals generate, the larger the crossover rate is, the easier the pattern of the old individual would be destroyed, then the new individuals generate would be faster. Excessive crossover rate may make more excellent individual mode destroyed, too small cross rate will delay the generation of new individuals, leading to prematurity and stagnation. The mutation rate is a key factor to decision algorithm to jump out of local optimal solution. The mutation rate is too small, which is not easy to generate a new mode structure, but mutation rate is so large to make GA the pure random search algorithm.

Adaptive genetic algorithm is hoping to find a more general adaptive crossover and mutation probability, to make the genetic algorithm more efficient. In the AGA, crossover and mutation probability is dynamic adjustment between the average fitness and the highest fitness of the population with the fitness of individual. They satisfy the following relationship:

$$
\begin{aligned}
& P_{X}=\left\{\begin{array}{c}
k_{1} \frac{\left(f_{\text {max }}-f^{\prime}\right)}{f_{\text {max }}-f_{\text {avg }}}, f^{\prime} \geq f_{\text {avg }} \\
k_{3}, f^{\prime}<f_{\text {avg }}
\end{array}\right. \\
& p_{m}=\left\{\begin{array}{c}
k_{2} \frac{\left(f_{\text {max }}-f^{\prime}\right)}{f_{\text {max }}-f_{\text {avg }}}, f^{\prime} \geq f_{\text {avg }} \\
k_{4}, f^{\prime}<f_{\text {avg }}
\end{array}\right.
\end{aligned}
$$

In the formula, $\mathrm{f}_{\max }$ is the group's largest fitness value; $f_{a v g}$ is the value of the average fitness of the population; $f^{\prime}$ is the larger of the two individuals to cross the fitness; $\mathrm{f}$ is the mutation individual's fitness value. Here, just set to take the value of $\mathrm{k}_{1}, \mathrm{k}_{2}, \mathrm{k}_{3}, \mathrm{k}_{4}$ (in $[0,1]$ value), $\mathrm{P}_{\mathrm{c}}$ and $\mathrm{P}_{\mathrm{m}}$ can be adaptively adjusted. According to AGA, When the individual's fitness is lower than the contemporary population average fitness, that the individual poor performance. If the individual is selected in the selection mechanism, its crossover and mutation rate will be large. When the individual fitness is close to the maximum fitness in contemporary populations, which will be regarded as the good performance of the individual, its excellent model would be retained as far as possible .Even if the individual is selected in the selection mechanism, also its lower crossover and mutation rate.

\section{Elitist strategy}

Elitist strategy is used for avoiding destroying the best individual per generation. Specifically described as follows: If the next generation of groups of individual fitness value is less than the current population of individual fitness value, the best individuals in the current groups or adaptation value is greater than the value of the next generation of the best individual fitness multiple individuals directly copied to the next generation, random substitution or substitution corresponding number of the worst individuals in the next generation groups. The elitist strategy ensures that the current best individual will not be destructed by crossover and mutation operations, It is a basic protection of groups converge to the optimal solution[4].

\section{IMPROVED ADAPTIVE GENETIC ALGORITHM}

\section{A. The improvement of the algorithm}

To improve the operating efficiency of the genetic algorithm, we made some improvements to the algorithm. In Adaptive Genetic algorithms, dynamically adjust the crossover and mutation probability is used to the individuals of different fitness. To ensure that the best individual in the population is not destroyed and poor individuals have a greater opportunity to participate in the process of crossover and mutation to improve the efficiency of evolution. Most adaptive genetic algorithm improvement strategies are focused on how to retain excellent individual, but excellent individual treatment, as well as poor individuals in the parent should be replaced and eliminated, to become the focus of our attention. First, in the simple binary encoding, due to the presence of the Hamming distance, the large Hamming distance resulting algorithm near even the most advantages it still difficult to find the most advantages. For example, 10000 is the optimal value, and the 01111 is suboptimal, lead to suboptimal individuals to the evolution of the best individual blocked, is reduced the generation probability of the advantages individual; Second, the poor individual does not necessarily represent the genes it carries completely does not help with evolution .

Hamming distance factors may produce suboptimal individual to the best individual in the evolutionary process, for the excellent individual to be determined, we decided to adopt the hybrid coding, it is binary coded and decimal encoding combined mode[5].However, due to the decimal encoding, individual variability will small ,We do not need to keep excellent individual low mutation rate in adaptive genetic algorithm to maintain its mutation probability constant, In this way, to avoid a large number of excellent individual inbreeding and stabilize falling into the local optimal solution, guarantee their diversity and improve the efficiency of the optimization, and allow excellent individual genes not to be completely destroyed.

The new adaptive crossover and mutation probability are shown in the following formula: 


$$
\begin{gathered}
P_{X}=\left\{\begin{array}{c}
k_{1} \frac{\left(f_{\max }-f^{\prime}\right)}{f_{\max }-f_{\text {avg }}}, f^{\prime} \geq f_{\text {avg }} \\
k_{3}, f^{\prime}<f_{\text {avg }}
\end{array}\right. \\
p_{m}=\left\{\begin{array}{l}
k_{2}, f^{\prime} \geq f_{\text {avg }} \\
k_{4}, f^{\prime}<f_{\text {avg }}
\end{array}\right.
\end{gathered}
$$

Wherein the variable definitions are the same as AGA, but $\mathrm{k}_{2}<\mathrm{k}_{4}$, to ensure that poor individuals have a greater probability of variation have excellent individual. For poor individuals, we introduce the second decision process, the crossover and mutation process with the probability of $100 \%$, If offspring individuals fitness $\mathrm{f}^{\prime}$ is more than the average fitness $f_{a v g}$, that its parent individuals holds the good genes, which could be retained as a potential individual. For the filial generation of individuals which is less than the average fitness, it is defined as no evolutionary potential individuals, which will be directly eliminated and replaced by the best individual in the previous generation with elitist strategy.

\section{B. Improved adaptive genetic algorithm process}

The improved adaptive genetic algorithm process:

1) Randomly generated initial population

2) Fitness of the individuals in the population according to the fitness function

3) Determine whether termination condition is satisfied, then go to 10)

4) Calculate $P_{c}, P_{m}$ according to the fitness

5) Select operations by roulette

6) Adaptive crossover operation

7) Decimal variability for the individuals greater than the average fitness, otherwise using binary variation

8) The judge new populations of poor individuals whether potential individual

9) Replace the non-potential individuals generate new populations Go 2)

10) Termination condition is met, the output statistics.

\section{Feasibility analysis algorithm improved}

In decimal variation, variability step has a direct impact on the ultimate effect of the variation. In the standard genetic algorithm, the mutation operator with variability of the real value is as follows:

$$
X^{\prime}=X \pm 0.5 L \Delta
$$

Where in $\Delta=\sum_{i=0}^{m=1} \frac{a(i)}{2^{i}}$, a the value 1 , take value 0 with probability (1-1/ m). Usually $\mathrm{m}$ $=20, \mathrm{~L}$ is the range of variables, $\mathrm{X}$ is a variation of the before variable, $\mathrm{X}$ ' is the variation after variation.

Here, in order to ensure the better individual variation as small as possible to come close to the optimum value, we use dynamic mutation operator, namely:

$$
X^{\prime}=X \pm \frac{\left(f_{\max }-f\right)}{2\left(f_{\max }-f_{\text {avg }}\right)} L \Delta
$$

Here $\mathrm{f}$ is the individual's fitness. In order to retain its excellent mode of individuals close to the maximum fitness, there are the following three theorems for binary variation operation:

Theorem 1: For any given $\mathrm{i} \in\{1,2, \cdots \cdots, \mathrm{n}\}, \mathrm{k} \in$ $\{1,2, \cdots \cdots, \mathrm{m}\}$, binary encoding individual $\mathrm{X}_{1}^{\mathrm{i}}$ the corresponding to the $\mathrm{k}$-th real number's smallest variation amount is $\frac{v_{k}-u_{k}}{2^{l}-1}$.

Theorem 1 show that for the binary encoding, the minimum variance can not be made arbitrarily small, It is subject to the limitations of the code lengths ,1 the longer the minimum variance is smaller, Therefore, for the binary coding, even in the vicinity of the optimal solution, mutation operation will not necessarily be able to search the optimal solution. Those provide a theoretical basis for the introduction of decimal coding for excellent individual.

Theorem 2: For any given $\mathrm{i} \in\{1,2, \cdots \cdots, \mathrm{n}\}, \mathrm{k} \in$ $\{1,2, \cdots \cdots, m\}$,binary encoding individual $x_{l}^{i}$ variation of only one, generate new individuals $x_{l}^{\prime}$, Set $s^{k}=\left|\Gamma\left(x_{l}^{i}, k\right)-\Gamma\left(x_{l}^{\prime}, k\right)\right|$, The maximum value of the $\mathrm{S}^{\mathrm{k}}$ is:

$$
s_{\max }^{k}=\frac{v_{k}-u_{k}}{2^{l}-1} \times 2^{l-1}
$$

The minimum value is:

$$
s_{\min }^{k}=\frac{v_{k}-u_{k}}{2^{l}-1}
$$

Theorem 3: If two individuals $X_{i}$ and $X_{j}$ for a given $k \in$ $\{1,2, \cdots \cdots, \mathrm{m}\}$ fulfill

$$
\Gamma\left(x^{i}, k\right)-\Gamma\left(x^{j}, k\right)=\frac{v_{k}-u_{k}}{2^{l}-1}\left(2^{p}-2^{q}\right)
$$

Wherein $\mathrm{p}, \mathrm{q} \in|1,2, \cdots \cdots, \quad \mathrm{l}-1|, \quad \mathrm{q}>\mathrm{p}, \quad \mathrm{X}_{\mathrm{i}}$ and $\mathrm{X}_{\mathrm{j}}$ by the cross after the new individual $\left(\mathrm{X}_{\mathrm{i}}\right)^{\prime}$ and $\left(\mathrm{X}_{\mathrm{j}}\right)^{\prime}$ collectively referred to as the $x^{\prime}$, to fulfill

$$
\Gamma\left(x^{i}, k\right)<\Gamma\left(x^{\prime}, k\right)<\Gamma\left(x^{j}, k\right)
$$

Its probability is not 0 .

Theorem 2 and 3 show that: the binary coded crossover and mutation operation, their offspring and parent individuals are not complete close necessarily, which provides the basis for us to retain a dominant gene poor individual.

\section{EXPERIMENT AND RESULT COMPARED}

In this paper, we choose the following test function:

$\mathrm{F}(\mathrm{x})=\mathrm{x} \sin (10 \pi \cdot \mathrm{x})+2.0, \quad(-1 \leqslant \mathrm{x} \leqslant 2)$ 
This function is limited to a maximum value slightly larger than 3.85,corresponding variable value of about 1.85.The experimental data for run 100 times, evolution algebra 150 generation, Parameter is set to the SGA crossover probability 0.80 ,Mutation probability 0.02 .

We can see from the data in the Table 1 , after 100 running respectively, the number of improved adaptive genetic algorithm converges will be slightly better, it shows the better robustness. And the optimal convergence effect will be gained, which is faster and better than AGA optimal solution.

\section{CONCLUSION}

Based on the original adaptive genetic algorithm, a new processing method is proposed for the high fitness individuals and poor individuals. We perform adaptive step mutation operation at individuals of high fitness hybrid coding, and for poor individuals, we introduce the judge conditions of the potential individual and non-potential individuals to ensure the accuracy of the elitist strategy. Experimental results show that this new algorithm could improve the efficiency of optimization and the speed of convergence, it is feasible and reliable.

\section{ACKNOWLEDGMENT}

This work has been supported by National Natural Science Foundation of China (No. 61074029), Liaoning Provincial Natural Science Foundation of China (No. 20102014), and Liaoning Provincial Science \& Technology Plan Project of China (No.2011216007).

\section{REFERENCES}

[1] J H Holland.Adaptation in Natural Artificial Systems [M]. MIT Press, 1976.

[2] Srinvas M,Patnaik L M.Adaptation Probabilities of Crossover and Mutation in Genetic Algorithms[J].IEEE Trans on Systems, Man and Cybernerics,1994;24(4):656-667.

[3] WANG Xiaoping,CAO Liming,Genetic algorithms-theory, application and software implementation[M].Xi'an: Xi'an Jiaotong University Press,2002.

[4] REN Ziwu,SAN Ye,Improved Adaptive Genetic Algorithm and its Application Research in Parameter Identification[J].Journal of System Simulation.2006,18(1):41-66.

[5] LI Bo,SHI Lin.Hybrid Encoding Genetic Algorithm and Its Application[J].Technology Square.2009,9:30-33.

TABLE I. COMPARISON OF THE PERFORMANCE OF THE THREE ALGORITHMS

\begin{tabular}{|l|l|l|l|l|}
\hline $\begin{array}{l}\text { Using } \\
\text { algorithm }\end{array}$ & $\begin{array}{l}\text { Convergence } \\
\text { Views }\end{array}$ & $\begin{array}{l}\text { Average } \\
\text { convergence } \\
\text { algebra }\end{array}$ & $\begin{array}{l}\text { Average } \\
\text { convergence value }\end{array}$ & Variable value \\
\hline SGA & 98 & 96 & 3.850269 & 1.850628 \\
\hline AGA & 98 & 40 & 3.850272 & 1.850562 \\
\hline $\begin{array}{l}\text { Improved } \\
\text { Algorithm }\end{array}$ & 99 & 38 & 3.850274 & 1.850546 \\
\hline
\end{tabular}

